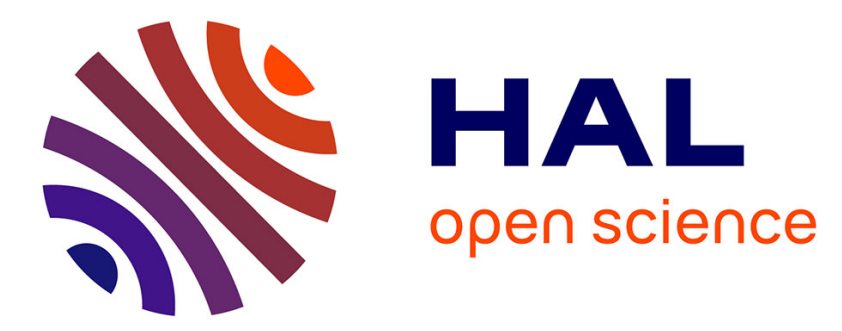

\title{
Instabilities produced by hot-wire heating below a free surface with emphasis on relationships between temporal and spatial dynamics
}

\author{
E. Ringuet, S. Meunier-Guttin-Cluzel, C. Rozé, G. Gouesbet
}

\section{- To cite this version:}

E. Ringuet, S. Meunier-Guttin-Cluzel, C. Rozé, G. Gouesbet. Instabilities produced by hot-wire heating below a free surface with emphasis on relationships between temporal and spatial dynamics. Journal de Physique II, 1994, 4 (8), pp.1243-1260. 10.1051/jp2:1994197 • jpa-00248040

\author{
HAL Id: jpa-00248040 \\ https://hal.science/jpa-00248040
}

Submitted on 1 Jan 1994

HAL is a multi-disciplinary open access archive for the deposit and dissemination of scientific research documents, whether they are published or not. The documents may come from teaching and research institutions in France or abroad, or from public or private research centers.
L'archive ouverte pluridisciplinaire HAL, est destinée au dépôt et à la diffusion de documents scientifiques de niveau recherche, publiés ou non, émanant des établissements d'enseignement et de recherche français ou étrangers, des laboratoires publics ou privés. 


\title{
Instabilities produced by hot-wire heating below a free surface with emphasis on relationships between temporal and spatial dynamics
}

\author{
E. Ringuet, S. Meunier-Guttin-Cluzel, C. Rozé and G. Gouesbet \\ L.E.S.P. $\left(^{*}\right)$, CORIA, Rouen INSA, B.P. 08, 76131 Mont Saint Aignan Cedex, France \\ (Received 22 December 1993, revised 16 April 1994, accepted 16 May 1994)
}

\begin{abstract}
Bifurcations up to the appearance of temporal chaos are observed when hot-wire heats below a free surface. New observations are reported, and some emphasis is laid on the relationships between temporal dynamics produced by signals averaged along the hot-wire and spatial behaviour of waves generated above it.
\end{abstract}

\section{Introduction.}

There is a growing interest in the study of instabilities generated by local heating below a free surface, leading to the observation of many nonlinear phenomena including developed chaos and intermittencies. Heating by a laser produces the so-called optical heartbeats or thermal lens oscillations which, beside allowing fundamental investigations in the framework of nonlinear dynamics (Refs. [1-3] and references therein) also leads to applications for concentration measurements of chemical species [4]. In another procedure to which this paper is devoted, heating is achieved by means of a hot-wire. This allows a better control of the distance between the heating location and the free surface. Temporal signals recorded at the ends of the hot-wire, thus averaging phenomena occurring along the wire, exhibited various kinds of behaviour including type II intermittency (Refs. [5-7] and references therein). On the other hand, spatial phenomena are involved in hot-wire experiments under the form of a train of waves propagating on the free surface, parallel to the wire, allowing an experimental study of $1 \mathrm{D}$ spatio-temporal chaos [8-10]. In particular, Vince and Dubois [8] examined the dynamics above a long $(L=60 \mathrm{~cm})$ hot-wire showing that the train of waves may exhibit several defects (sources and sinks) which however eventually relax to a simpler situation with 0 or 1 defect. No defect is observed when the distance between the wire and the surface is large. In such a case, spatio-temporal disorganization of the train of waves may be observed. Furthermore, in this case, the waves have a significant spatial extension perpendicular to the wire and may be

(*) U.R.A. CNRS 230 . 
interacting with confinement walls. In the present paper, hot-wire experiments are carried out with a smaller wire $(6 \mathrm{~cm})$, allowing a better study of temporal signals averaging along the wire and spatial phenomena, without any lateral confinement effect. Also, a very simple model enables us to predict under which conditions nonperiodic phenomena may be expected in the case when no spatial defect occurs.

The paper is organized as follows. Section 2 briefly describes the experimental set-up and the primary instability which is characterized as a supercritical Hopf bifurcation. Section 3 introduces a model relating spatial wave patterns and temporal signals in the absence of any spatial defect. Experiments are reported to demonstrate the pertinence of the model. Section 4 presents a zoo of temporal behaviours examplifying the richness of the system. In the case of quasiperiodicity, temporal behaviour may easily be related to the spatial organization of the wave pattern. Section 5 is devoted to the coexistence of attractors. In sections 4 and 5 , the observed behaviours correspond to the existence of one spatial defect. In all these sections, the relations between temporal signals and spatial patterns are discussed whenever possible. Section 6 is a conclusion.

\section{Experimental set-up and the primary instability.}

2.1 EXPERIMENTAL SET-UP. - The experimental set-up is similar to the one described by Rozé et al. [6], but with two modifications : (i) the wire length is $6 \mathrm{~cm}$ instead of 3 and (ii) it is current controlled instead of being temperature controlled.

The fluid under study is a silicon oil (Rhodorsil 47V10) with a Prandtl number equal to 130. The oil is contained in a tank $\left(17 \times 12 \times 10 \mathrm{~cm}^{3}\right)$ whose walls are kept at a constant temperature $\left(T_{0} \approx 25^{\circ} \mathrm{C}\right)$ by using thermostated water. This tank is located in a thermostated box which keeps the ambient temperature at $T_{0}$ within $0.05 \mathrm{~K}$. The fluid is heated by a platinum wire with a diameter equal to $20 \mu \mathrm{m}$. Its resistance can be written as :

$$
R(T)=R_{0}\left[1+\alpha\left(T-T_{0}\right)\right]
$$

where $R_{0} \approx 18 \Omega$ is the wire resistance at the reference temperature $T_{0}, \alpha$ is the temperature coefficient of the platinum $\left(\alpha=3.9 \times 10^{-3} \mathrm{~K}^{-1}\right)$ and $T$ is the spatial average of the wire temperature. The ratio of the wire length $L$ over the wire diameter is large enough to ensure that the temperature profile is essentially constant along the wire in the steady state, in such a way that edge effects are assumed to be negligible [11, 12].

The wire is soldered at the top of two thin strips and is tight horizontally (Fig. 1) below the free surface of the fluid. Horizontality is adjusted by making the wire and its image (formed by reflexion on the free surface) parallel when the wire is very close to the surface. The distance between the surface and the wire is taken to be zero when the wire and its image are blended. The vertical position of the wire is adjusted by means of a micrometric pedestal described in reference [13].

The wire is supplied with a constant current $(I)$ which is controlled by using a microcomputer. The Joule effect resulting from the current induces the heating of both the wire and the fluid surrounding the wire. By using a second micro-computer, the wire voltage is recorded with a sampling frequency which may be adjusted between 20 and $400 \mathrm{~Hz}$. Time series of 160000 consecutive values may be recorded.

The wire voltage $U$ is related to the spatially average temperature $T$ by Ohm's law :

$$
U=R_{0}\left[1+\alpha\left(T-T_{0}\right)\right] l .
$$

The dynamics depends on two control parameters (i) the distance $d$ between the wire and the free surface and (ii) the intensity $I$ supplied to the wire. 


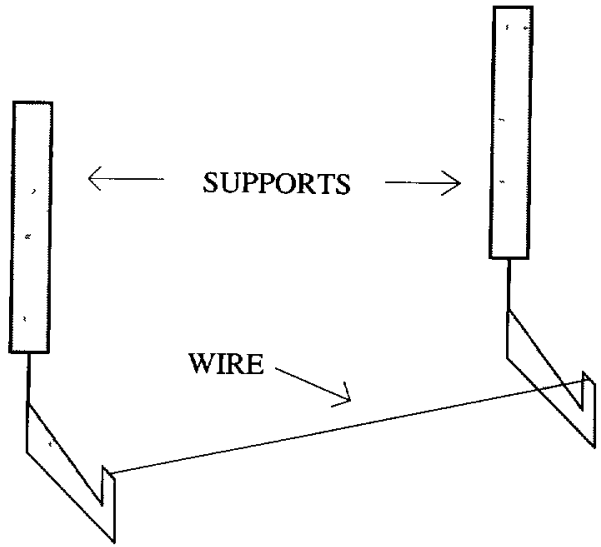

Fig. 1. - Schematic representation of the supports of the wire.

2.2 THE PRIMARY INSTABILITY. - For a fixed distance $d$ and for a low temperature $T$, the system is steady. The free surface is deformed, producing a crest or a trough depending on whether $d$ is large or small. A double convection roll is established along the wire (see for instance numerical simulations in Ref. [14]). When $T$ is increased, the system may undertake a supercritical Hopf bifurcation [6], leading to oscillatory behaviour, if $d$ is not too large. Waves then appear on the free surface, propagating parallel to the wire. Some characteristics of these phenomena may be understood, and even quantitatively predicted, by a simple model relying on the existence of two characteristic times (i) a heat transport time corresponding to the time required for the heat transport between the wire and the free surface and (ii) a Marangoni time corresponding to the disruption of temperature gradients at the surface [15].

For measuring the critical temperature $T_{\mathrm{c}}$ (critical intensity $I_{\mathrm{c}}$ ) at which the system becomes unstable, the intensity is gradually increased for a fixed $d$ up to the appearance of oscillatory behaviour. The onset of oscillatory motion may be detected (i) optically, by illuminating the surface with an expanded laser beam and observing the reflected light on a screen or (ii) electrically, by examining the wire voltage time series which becomes periodic at the onset [6].

The critical intensity $I_{\mathrm{c}}$ is displayed versus $d$ in figure $2 \mathrm{a}$, the points below and above the curve corresponding to steady and oscillatory behaviour, respectively. Periods $F_{\mathrm{c}}^{-1}$ at the onset versus $d$ are displayed in figure $2 \mathrm{~b}$. Increasing $d$ from zero, periods are first small $(0.1 \mathrm{~s}$ for
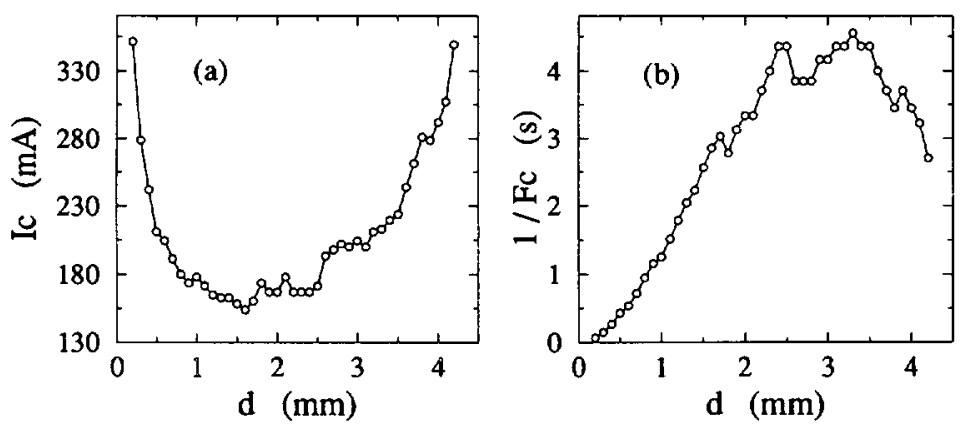

Fig. 2. - The onset of oscillatory motion occurs for different values $I_{\mathrm{c}}$ of the intensity depending on the distance $d(\mathrm{a})$, with a certain period $T_{\mathrm{c}}=1 / F_{\mathrm{c}}$ (b). 
$d=0.2 \mathrm{~mm}$ ), then increase in a non-regular way up to $4.5 \mathrm{~s}$ and eventually decrease. These results are similar to those previously described $[6,8,13]$. The critical wavelength of the propagating waves is about 10 times the distance $d[6,8]$. Relying on the experimental determination of the disruption relation of these waves, it is claimed that they are neither pure capillary nor pure gravity waves [6]. Their exact nature is therefore still unknown. Let us however mention that a connection with hydrothermal surface-wave instability disturbances governed by the Kuramoto-Sivashinsky equation has been recently discussed [16]. When the horizontality of the wire is perfectly well adjusted, there is no privileged direction of propagation.

\section{Spatial wave pattern and temporal signals : model and experiments.}

In the steady state, the temperature is constant along the wire and can readily be evaluated by measuring $U$ (Eq. (2)). When waves propagate along the wire, it is assumed that the local temperature at location $x$ along the wire and at time $t, T(x, t)$ is slaved to the local state of the waves, i.e.

$$
T(x, t)=f(x-v t)
$$

where $v$ is the wave velocity. This expression is allowed by the fact that waves propagate without any deformation. Also, equation (3) assumes the absence of any spatial defect in the wave train. When the phenomena are periodic, the unknown function $f$ may be expanded as a Fourier series reading :

$$
T(x, t)=\sum_{n=1}^{\infty}\left\{a_{n} \cos \left[\frac{2 \pi n}{\lambda}(x-v t)\right]+b_{n} \sin \left[\frac{2 \pi n}{\lambda}(x-v t)\right]\right\}
$$

where $\lambda^{\prime}$ is the spatial period (wavelength) of the wave, and the constant term has been dismissed.

The spatial average of the temperature along the length $L$ of the wire then reads :

$$
\bar{T}(t)=\frac{1}{L} \int_{0}^{L} T(x, t) \mathrm{d} x
$$

which becomes :

$$
\bar{T}(t)=\frac{\lambda}{2 \pi L} \sum_{n=1}^{\infty}\left\{C_{\cos }^{n} \cos \left(\frac{2 \pi n}{\lambda} v t\right)-C_{\sin }^{n} \sin \left(\frac{2 \pi n}{\lambda} v t\right)\right\}
$$

where :

$$
\begin{aligned}
& C_{\mathrm{co}}^{n}=\frac{a_{n}}{n} \sin \left(2 \pi n \frac{L}{\lambda}\right)+\frac{b_{n}}{n}\left[1-\cos \left(2 \pi n \frac{L}{\lambda}\right)\right] \\
& C_{\mathrm{\iota n}}^{n}=\frac{a_{n}}{n}\left[1-\cos \left(2 \pi n \frac{L}{\lambda}\right)\right]-\frac{b_{n}}{n} \sin \left(2 \pi n \frac{L}{\lambda}\right)
\end{aligned}
$$

which also gives the form of the time signal $U(t)$ which is proportional to $\bar{T}(t)$, with the constant term dismissed.

If the number of waves $L / \lambda$ along the wire is an integer $p$, then $C_{\text {cos }}^{n}=C_{\text {sin }}^{n}=0$ and $\bar{T}(t)$ is also zero (or actually a constant). On the other hand, if $L / \lambda=(p+1 / 2)$, then the coefficients are zero if $n$ is even. In such a case. the spectrum of the signal will not exhibit peaks at frequencies $2 F_{0}, 4 F_{0}$. where $F_{0}$ is the fundamental frequency. 
Averaging $(\bar{T})^{2}$ over a time period of the signal $\bar{T}(t)$, we obtain the total power of the signal $P(L / \lambda)$ which reads :

$$
P=\frac{1}{2}\left(\frac{\lambda}{2 \pi L}\right)^{2} \sum_{n=1}^{\infty}\left\{\left(C_{\mathrm{cos}}^{n}\right)^{2}+\left(C_{\mathrm{sin}}^{n}\right)^{2}\right\}
$$

The function $f(x-v t)$ being unknown, it is tentatively assumed that only $a_{1}$ is different from zero in relation (4), leading to :

$$
P=2 a_{1}^{2}\left(\frac{\lambda}{2 \pi L}\right)^{2} \sin ^{2}\left(\frac{\pi L}{\lambda}\right) .
$$

This result is displayed as $A=\sqrt{P}$ versus $\lambda / L$ in figure 3 . In the framework of this simple model, the dimensionless ratio $\lambda / L$ appears to be a pertinent parameter to organize the results in the absence of any spatial defect. Let us remark that the control parameters are $I$ and $d$ (Sect. 2.1). For $I$, given, $\lambda$ depends on $d$ as demonstrated in references [6, 8]. Therefore, parameters might be taken as the control parameter $I$ and the model parameter $(\lambda / L)$. The control parameter $I$ would modify the function $f(\lambda-v t)$ of equation (3). This function being assumed to be unknown in the above simple model, $I$ is no longer a relevant parameter in this restricted framework.

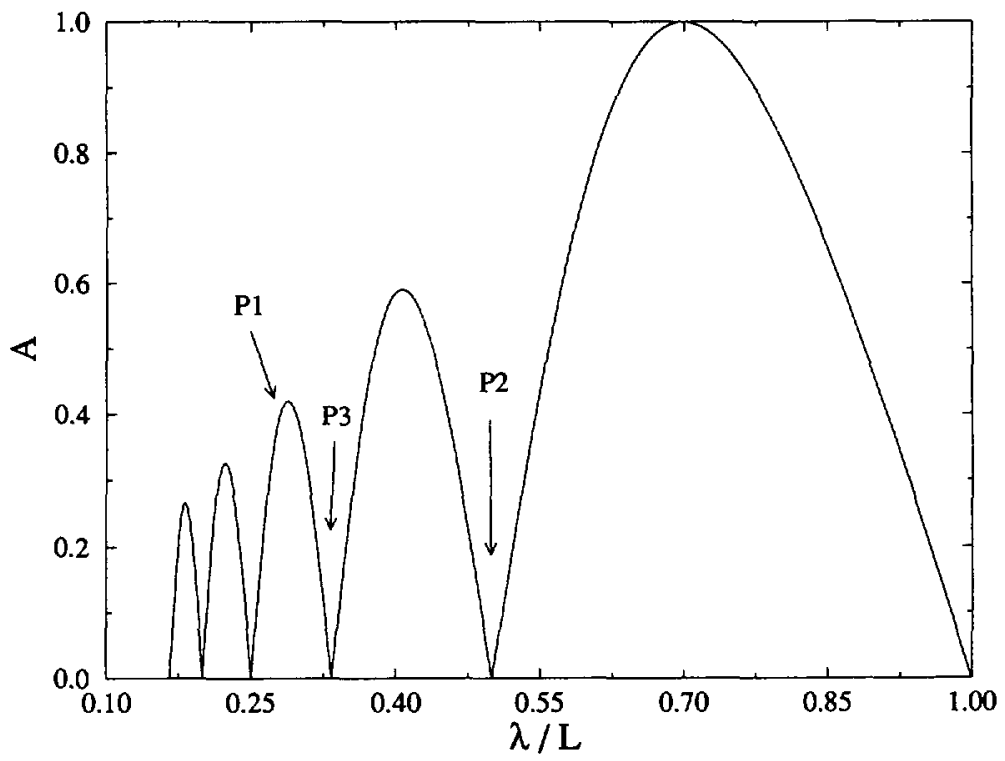

Fig. 3. $-A=f(\lambda)$ : simple model.

Experimentally, the total power of the signal is extracted by computing the power spectrum and summing power spectral densities over all frequencies. For a fixed current $I$, the wavelength $\lambda$ is adjusted by changing the distance $d, \lambda$ decreasing when $d$ decreases. In reference [6], the wavelength $\lambda$ has been measured by using a laser reflection technique, the reflected image being studied by a photodiode array. In the present paper, we measured the propagation time $t_{\mathrm{p}}$ of a wave over the length $L$ of the wire, yielding the wave velocity $v=L / t_{p}$ and the characteristic frequency $F$ yielding the period $T$, leading to $\lambda=L T / t_{\mathrm{p}}$. An example of experimental results is displayed as $A$ versus $d$, for $I=250 \mathrm{~mA}$, in figure 4 , 


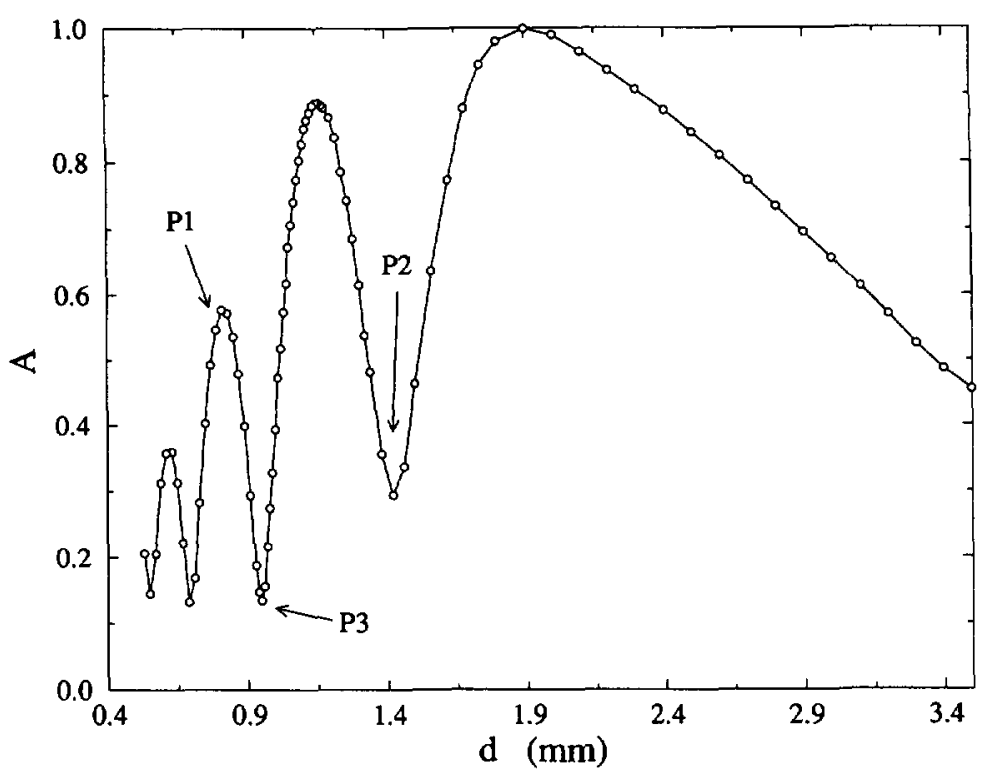

Fig. 4. $-A=f(d)$ : experımental results for $I=250 \mathrm{~mA}$.

exhibiting the pertinence of the model (Fig. 3). In particular, for the right bump in which $d$ evolves from 3.4 to $1.4 \mathrm{~mm}, \lambda / L$ evolves from about 1 to 0.5 . For the next bump, $\lambda / L$ evolves from 0.5 to $1 / 3$. During these experiments, the direction of propagation of the waves remained unchanged. This situation can be sketchily represented by the following diagram : $\rightarrow \rightarrow \rightarrow \rightarrow$.

Figure 5 displays a time signal and its power spectrum for $L / \lambda=3.5 \pm 0.1$, illustrating the simplicity of the dynamics when $L / \lambda$ is of the form $(p+1 / 2), p$ being an integer. Although all harmonics are present, the amplitude of the peak $2 F_{0}$, for instance, is much smaller than the
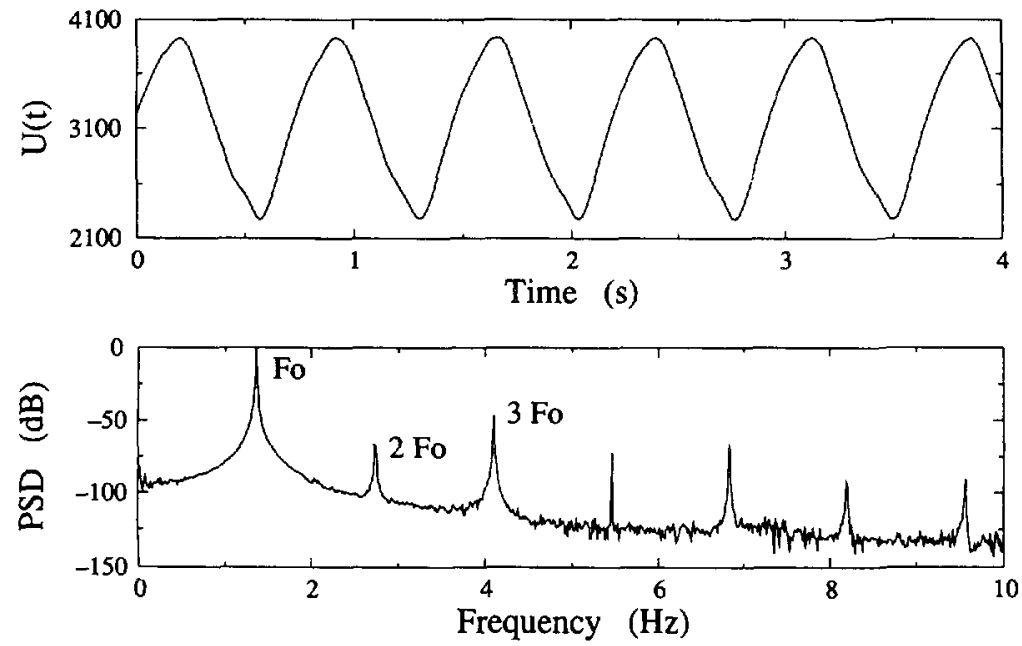

Fig. 5. - Time signal and power spectrum density (PSD) obtained for $L / \lambda=3.5 \pm 0.1 \quad(d=0.74 \mathrm{~mm}$ and $I=280 \mathrm{~mA}$ ). $U$ is given in arbitrary unit. 
amplitude of the peak $3 F_{0}$. This situation corresponding to $\lambda / L \approx 0.29$ is indicated by the label P1 in figures 3 and 4 . The signal power being large, the Signal/Noise Ratio (SNR) is very large too. Conversely, figure 6 displays a time signal and its power spectrum for $L / \lambda=2.0 \pm 0.1$, which is very different from $(p+1 / 2), p$ integer (label P2 in Figs. 3 and 4$)$. The signal is more complicated, even harmonics are bigger and the SNR is significantly deteriorated.
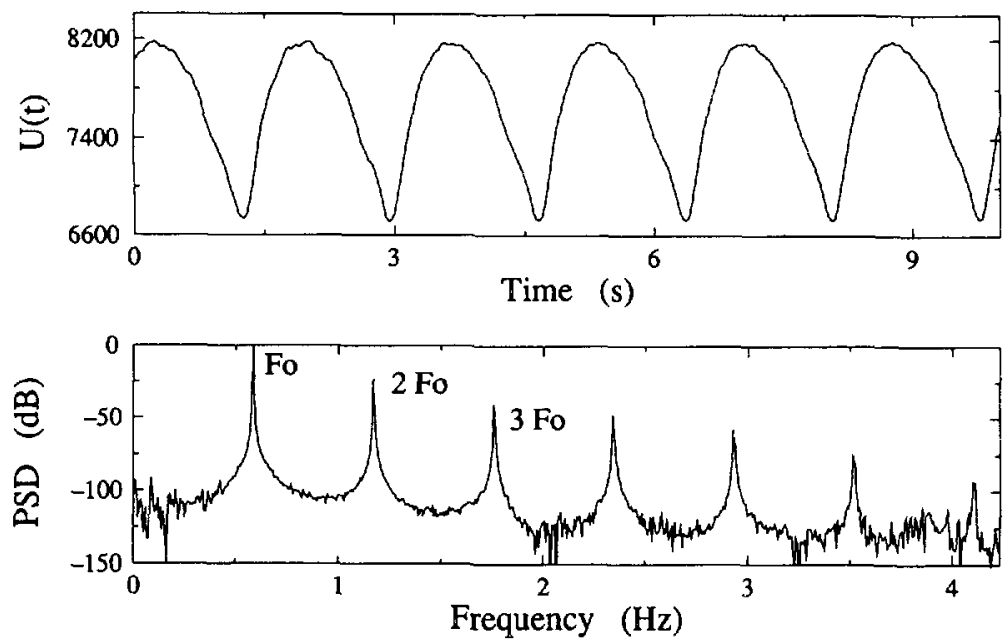

Fig. 6. - Time signal and power spectrum density (PSD) obtained for $L / \lambda=2.0 \pm 0.1(d=1.45 \mathrm{~mm}$ and $I=250 \mathrm{~mA}$ ).

Actually, the states in which the total power is a minimum are special states. They mark the transition between two bumps, one for which $L / \lambda$ reads $N+r, 0<r<1, N$ integer $>0$, and another one for which $L / \lambda$ reads $N+r+1$. According to the aforementioned idealized simple model, the power minima are 0 (Fig. 3). Actually they are not, probably partially due to the fact that such minima are very difficult to detect experimentally (Fig. 4). In the absence of any defect, a periodic behaviour is observed in the domains corresponding to the bumps and nonperiodic behaviour is observed near the minima. On the other hand, in the presence of a defect, non-periodic behaviour may be observed up to $(\lambda / L)$ about equal 0.5 , i.e. when there are at least two waves on the wire. With two waves and no defect separating the two waves, only periodic behaviour has been observed.

As an example of non-periodic behaviour, let us consider the time series and its associated power spectrum in figure 7 for $L / \lambda=3.0 \pm 0.1$ (label P3 in Figs. 3 and 4). The power spectrum has been evaluated by averaging 10 spectra built from 8192 data points. This state has been characterized as being chaotic, in the spectrum, the chaos leads to a broadband contribution at frequencies smaller than the fundamental frequency $\left(F_{0}\right)$. There, with waves propagating in the same direction, the observed simple periodic behaviour corresponds to a case when there are $(p+1 / 2)$ individual waves along the wire, $p$ being an integer, while, conversely, more complex behaviour may be expected when there is an integer number of waves along the wire. The physical reason why it is so remains to be identified.

In order to discuss the relation between temporal signals and spatial patterns more extensively, the free surface is filmed by means of a thermal camera, allowing us to record the time evolution of the temperature field. To match the small sampling frequency of the camera (25 images per second), experiments must be carried out in a small frequency regime such as 


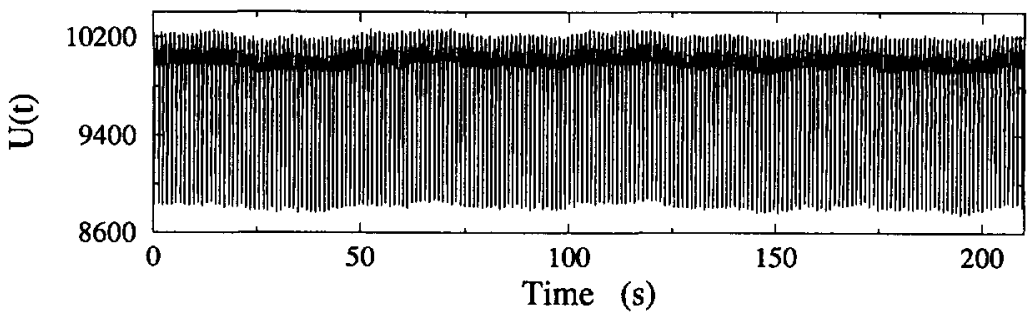

(a)

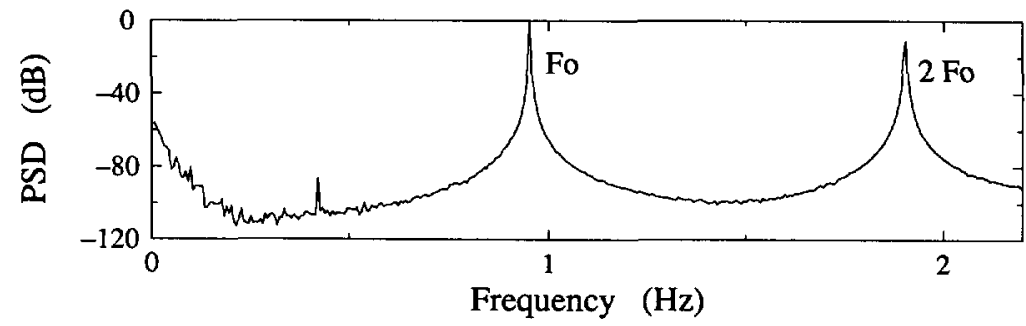

(b)

Fig. 7. - Chaotic behaviour observed for a minimum of the total power for $L / \lambda=3.0 \pm 0.1$ $(d=1.0 \mathrm{~mm}$ and $I=260 \mathrm{~mA})$.

for $d=3.5 \mathrm{~mm}, I=260 \mathrm{~mA}$. In this case for which the state is periodic, the temperature of a point located on the surface, on the vertical above the wire, has been recorded during a few periods leading to the temperature profile of an individual wave displayed in figure 8 . This temperature profile owns a strong similarity with the surface deformation profile published in reference [6], figure 15 . The profile in figure 8 may be viewed as ancillation made of two steps : (i) an increase of the temperature for $x / \lambda$ ranging from 1 to 0.4 , due to the arrival of heat from the heating source followed by (ii) a decrease due to the Marangoni disruption of the temperature gradients.

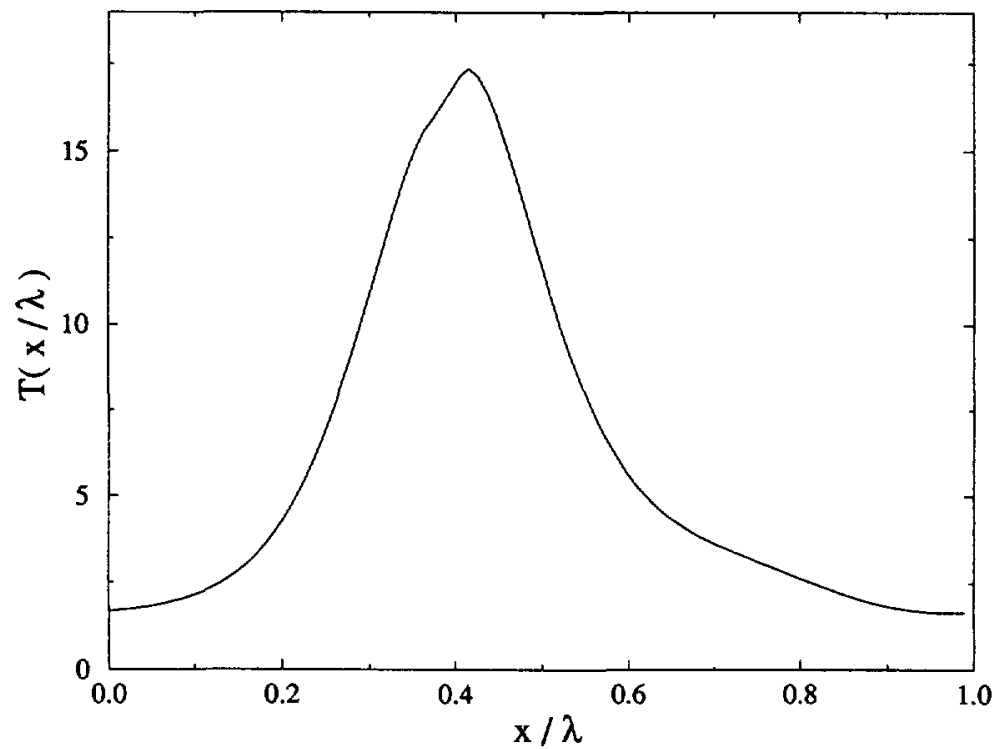

Fig. 8. - Spatial temperature profile of the waves observed when the wire is far from the surface $(d=3.5 \mathrm{~mm}) . T$ is given in arbitrary unit. 
The question then arises to know to which extent the information gained by measuring the average temperature $\bar{T}(t)$ from $U(t)$ is equivalent to that gained by measuring a local temperature $T\left(x_{0}, t\right)$. In some cases at least, the answer is positive. First, this is the case for the trivial situation of the basic stable state in which both $\bar{T}$ and $T\left(x_{0}\right), \forall x_{0}$, do not depend on $t$. Second, this is also the case for a periodic state in which both $\bar{T}(t)$ and $T\left(x_{0}, t\right)$ are periodic in time, with, furthermore, the same period. Next, we consider the case of a chaotic signal in which the spatial pattern exhibits one sink where counter-propagating waves encounter and disappear, according to the diagram : $\rightarrow \rightarrow \rightarrow \leftarrow$. It then appears that the temporal chaos of the time signal is correlated with a spatial chaotic behaviour of the sink whose position is observed to be chaotically wandering. In figure 9 this statement is illustrated by comparing several time signals (i) the average temperature $\bar{T}(t)$ (a) (ii) a local temperature in a small domain of the surface in which the sink motion is confined (b) and (iii) a local temperature recorded far away from the sink (c). The strong similarity between figures $9 \mathrm{a}$ and $9 \mathrm{~b}$, showing six synchronized groups of oscillations (separated by vertical lines), visually illustrates the correlation between the chaotic average temperature $\bar{T}(t)$ and the chaotic spatial motion of the sink. On the other hand, the comparison between figures $9 \mathrm{~b}$ and $9 \mathrm{c}$ indicates that the spatial chaotic behaviour associated with the sink is indeed strongly localized. As a whole, the system remains strongly organized in space and the information of the sink wandering is then transferred to the average temperature in an easily way.

Therefore, in spite of the apparent complexity of the system which is open and involves propagating waves, the spatial auto-confinement of defects allows us to characterize states with a small number of degrees of freedom by using the tools of nonlinear dynamics.

\section{A zoo of temporal behaviours.}

All the states analyzed in this section are generated by the presence of a single sink point, i.e. the simple model discussed in section 3 is no longer relevant. The process of production of the sink will be discussed in section 5. Yet, even with a single sink point, many different kinds of behaviour may appear, simple or complex. Typical transient times needed to reach the

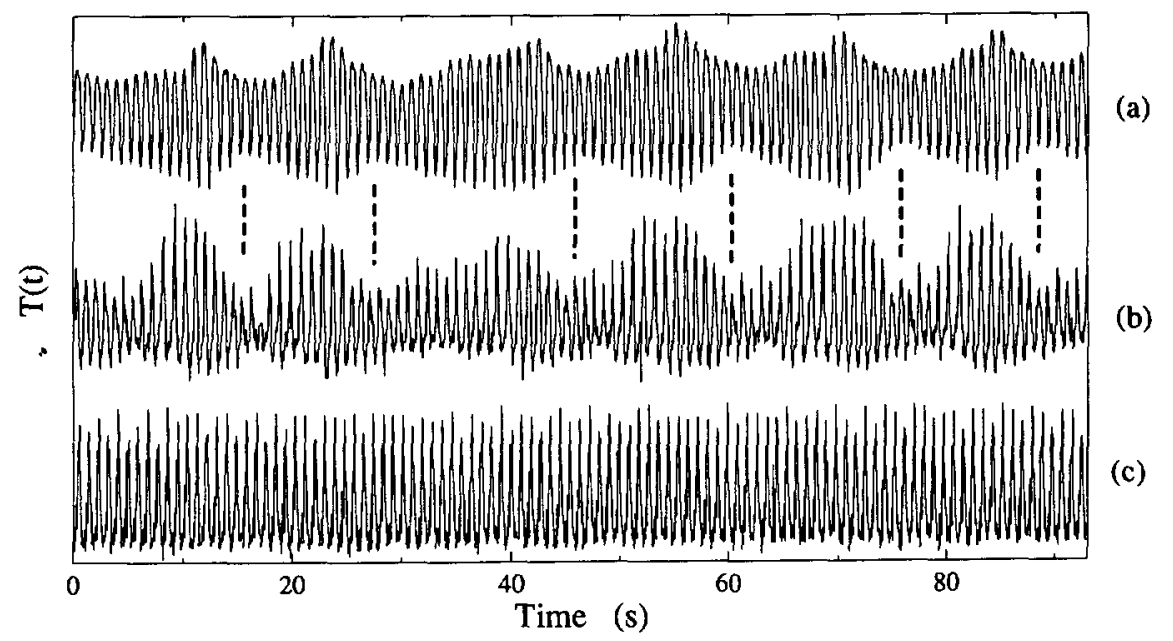

Fig. 9. - Comparison between averaged along the wire and local temperatures at the free surface $(d=0.9$ and $I=280 \mathrm{~mA})$. 
described asymptotic states are short, at worst a few seconds. The obtained states are very stable. They may be observed without detectable modifications during runs lasting several hours. Reproducibility is very good when the ratio $\Delta A / \Delta d$ is small in figure 4 , in which $\Delta A$ is the modification of $A$ induced by the variation $\Delta d$ of $d$. In particular, this is the case for the first two bumps (starting from the right) down to $d \approx 1 \mathrm{~mm}$.

4.1 QUASIPERIODICITY. - The sink point separates two domains in which waves propagate in opposite directions. Wave frequencies in each domain are found to be slightly different. Two frequencies are therefore present in the system, leading to quasiperiodicity if these frequencies are incommensurable. The difference in frequencies also implies that the sink point, i.e., the point where the waves meet, must move.

A wire voltage time series is displayed in figure 10, its power spectrum (Fig. 17b) exhibits two incommensurable frequencies $F_{1}=2.051 \mathrm{~Hz}$ and $F_{2}=2.199 \mathrm{~Hz}\left(\Delta F_{1}= \pm 0.003 \mathrm{~Hz}\right)$. The attractor is a 2 -torus which may be reconstructed in a $3 \mathrm{D}$-phase space using the time-delay method. The intersection of the reconstructed torus with a Poincaré section is displayed in figure 11. Frequency incommensurability implies that all the surface of the torus is visited.

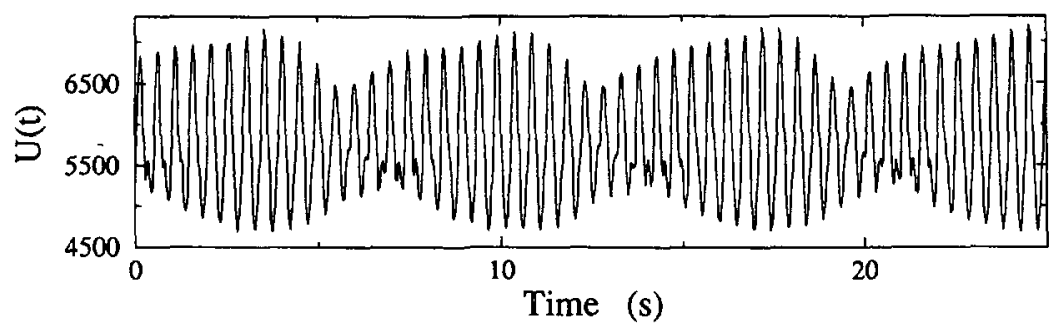

Fig. 10. - Quasiperiodic behaviour of $U(t)(d=0.62 \mathrm{~mm}$ and $I=260 \mathrm{~mA})$.

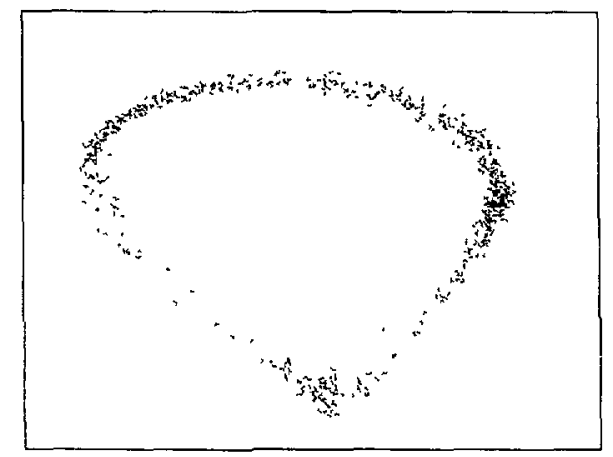

Fig. 11. - Poincaré section of the 2-torus.

Let $P$, be the successive points in the Poincaré section of figure 11 and $B$ be the barycenter of the set of points, $i=0, ., N$. The phase angle $\phi$, of points $P_{1}, i=1, \ldots, N$ is defined as the angle $\left(P_{0} B P_{1}\right)$. From the $\phi_{1}$ 's, taken in the range $(0,2 \pi)$ and then normalized in the range $(0,1)$, we obtain the first return map $\phi_{1+1}=f\left(\phi_{1}\right)$ displayed in figure 12. After fitting the data by parts, by using a least squares method associated with a SVD (Singular Value 


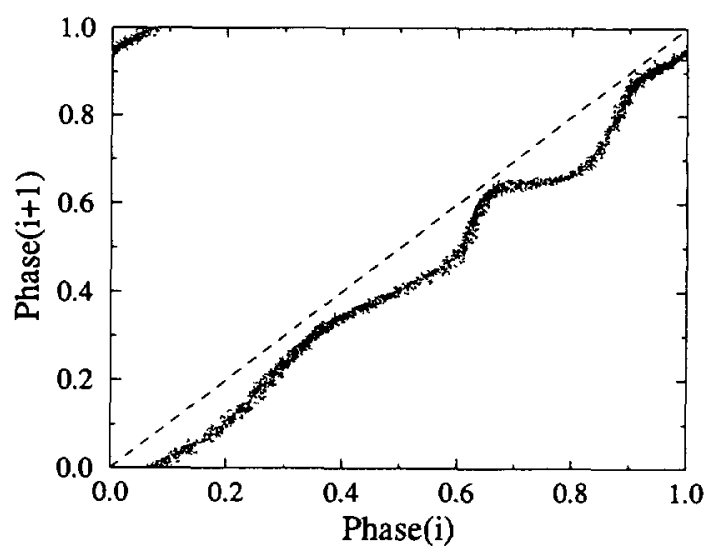

Fig. 12. - First return map for the phase angle defined in the Poincaré section.

Decomposition) algorithm, the slope of the curve may be evaluated at each point $\phi_{i}$. The Lyapunov exponent of the map can be computed afterward by using [17]:

$$
\lambda=\frac{1}{N} \sum_{t=1}^{N} \ln \left|f^{\prime}\left(\phi_{i}\right)\right| .
$$

This Lyapunov exponent, which is also the greatest Lyapunov exponent of the time series, is then found to be $\lambda=-0.063 \pm 0.005$, indicating a nonchaotic behaviour.

4.2 CHAOTIC PHASE INTERMITTENCY. - The temporal evolution is displayed in figure 13, its careful examination shows that the groups of oscillations are of different lengths. The spectrum exhibits two frequencies $F_{1}=1.024 \mathrm{~Hz}$ and $F_{2}=1.106 \mathrm{~Hz}$ near a frequency locking of ratio $F_{1} / F_{2}=1$. However in contrast with the previous case where two incommensurable frequencies led to quasiperiodicity, we are here faced with chaotic phase intermittency. The only other experimental observation of phase intermittency we are aware of has been obtained in Rayleigh-Bénard convection by Bergé and Dubois [18].

To illustrate this phenomenon, figure 14 displays a few points in a Poincaré section of a 3Dtime delay reconstruction. The points are numbered in chronological order. There is a regular drift from points 1 to 10 followed by a slowing down for points 11 to 14 , produced by the proximity of the frequency locking, and then by a large change from point 14 to 15 . This behaviour is characteristic of the phase intermittency phenomenon.

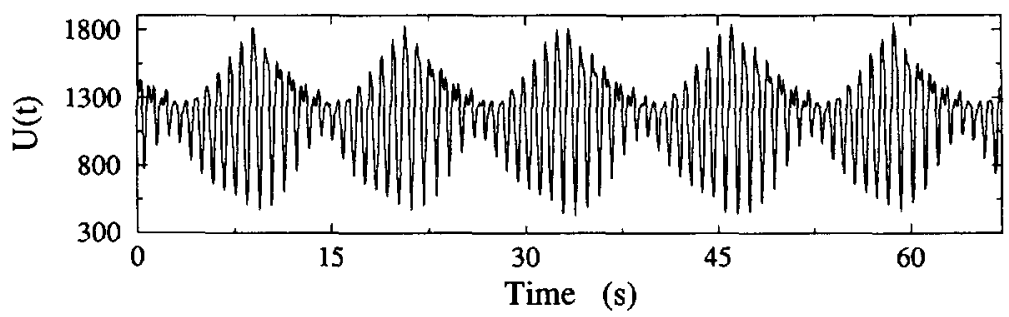

Fig. 13. - Chaotic time series of phase intermittency. The longest group of oscullations is in the middle $(d=1.04 \mathrm{~mm}$ and $I=290 \mathrm{~mA})$. 


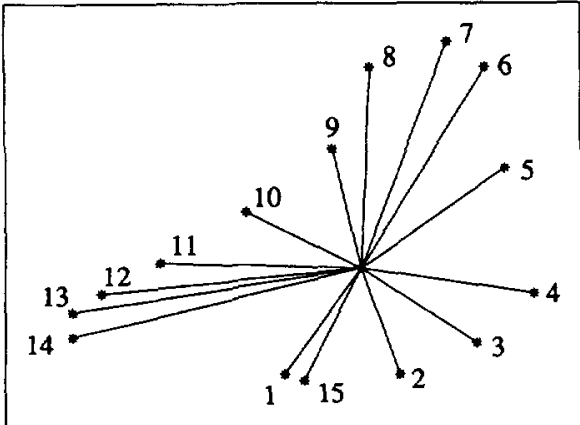

Fig. 14. - Illustration of the intermittent variation of the phase angle in the Poincare section.

The first return map $\phi_{1+1}=f\left(\phi_{1}\right)$, is shown in figure 15, exhibiting two channels nearly tangent to the bisector, associated with the intermittency. This map is more complicated than in the previous case (Fig. 12) with the result that the map Lyapunov exponent is difficult to extract by using relation (11). Therefore, the greatest Lyapunov exponent has been evaluated by studying the temporal evolution in a reconstructed phase space, according to an algorithm developed by Rosenstein et al. [19], leading to $\lambda \approx 0.20 \mathrm{~s}^{-1}$. This positive value indicates a chaotic behaviour. The probability distribution of the duration of one apparent rotation in the Poincaré section is displayed in figure 16 . The presence of chaos is also confirmed by the time series power spectrum (Fig. 17a) which exhibits a noisy background, to be compared with the power spectrum of the previously discussed quasiperiodic signal in figure $17 \mathrm{~b}$.

4.3 TYPE-I PSEUDO-INTERMITTENCY, - Type-I intermittency is generated by the loss of stability of a limit cycle when a real eigenvalue of the linearized Poincare map crosses the unit circle at $(+1)$ and leads to time signals made of laminar phases interrupted by chaotic bursts [20]. This kind of behaviour has been previously observed in hot-wire experiments [5-

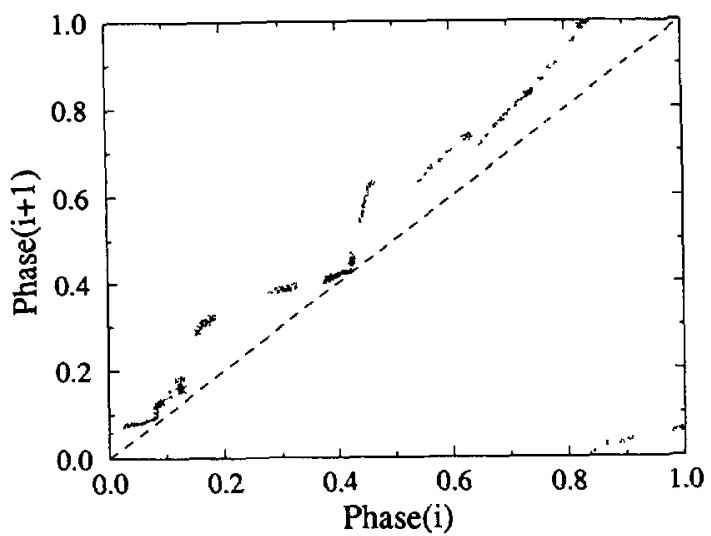

Fig. 15 .

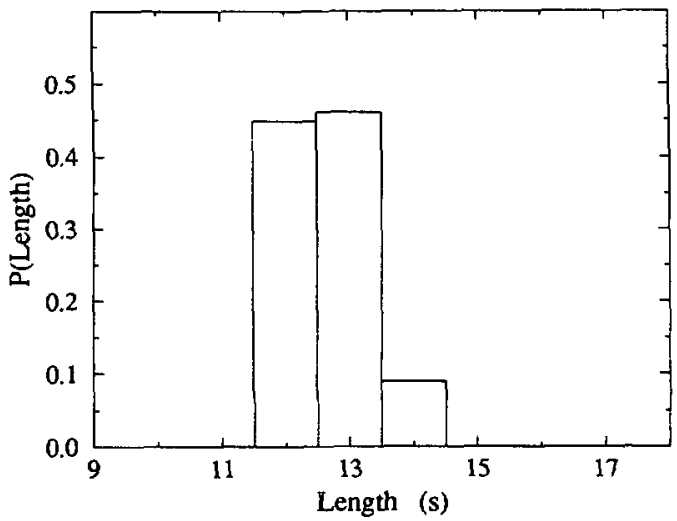

Fig. 16 .

Fig. 15. - First return map for the phase angle.

Fig. 16. - Relative probability distribution of the time required for one apparent rotation in the Poincaré section. 


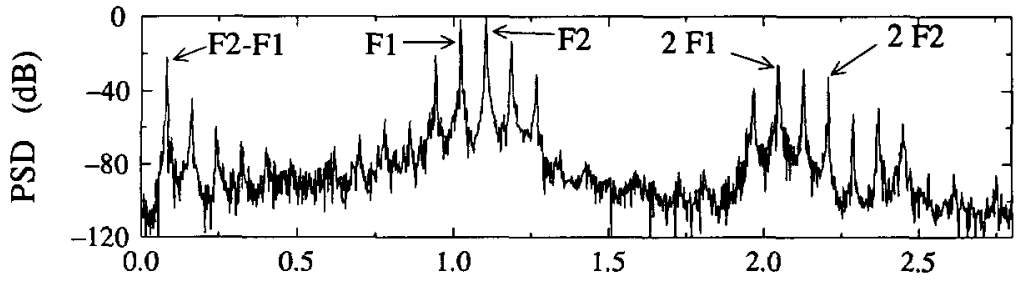

(a)

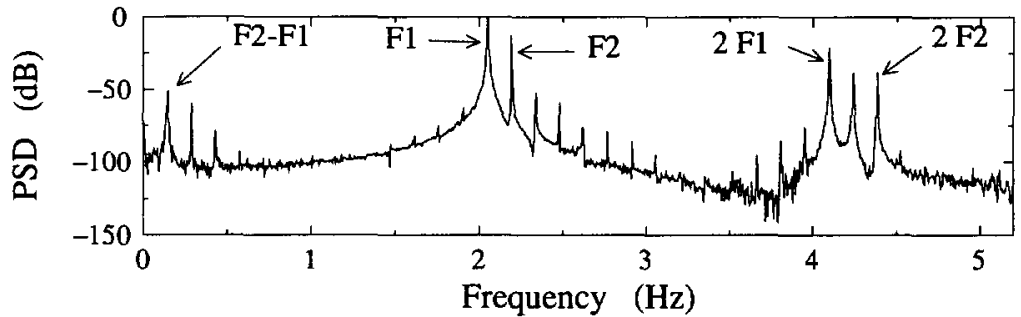

(b)

Fig. 17. - Comparison between two power spectra : (a) chaotic phase intermittency, (b) quasiperiodicity.

21]. In this section, we present a case sharing many common points with type-I intermittency stricto sensu except for the presence of hysteresis (discussed in the next section), hence the name of type-I pseudo-intermittency.

By varying the distance $d$, we observed a bifurcation from a periodic state to the attractor displayed in figure 18. The dark region is reminiscent of the old limit cycle which lost its stability. The first return map based on the successive maxima of the signal exhibits a channel near the bisector as in theoretical models (Fig. 19). The probability distribution $P(L)$ giving the probability $P$ of observing a laminar phase of length $L$ is shown in figure 20 . The maximal probability is for $L \approx 40 \mathrm{~s}$. For larger $L ' \mathrm{~s}$, the probability decreases fast. Moreover, there is a second significant peak of probability for $L \approx 10 \mathrm{~s}$. Such characteristics are in fair agreement with theoretical results [22].

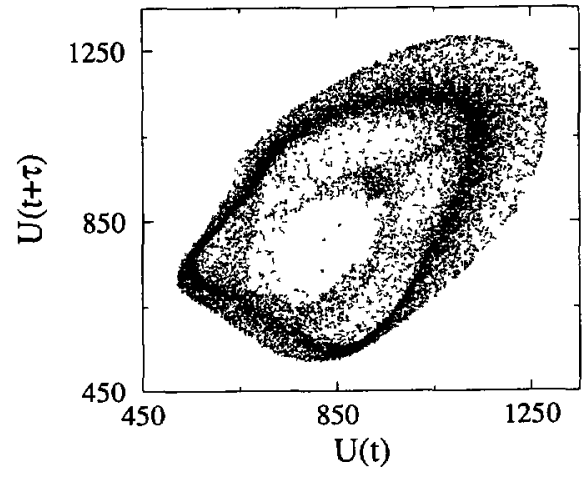

Fig. 18 .

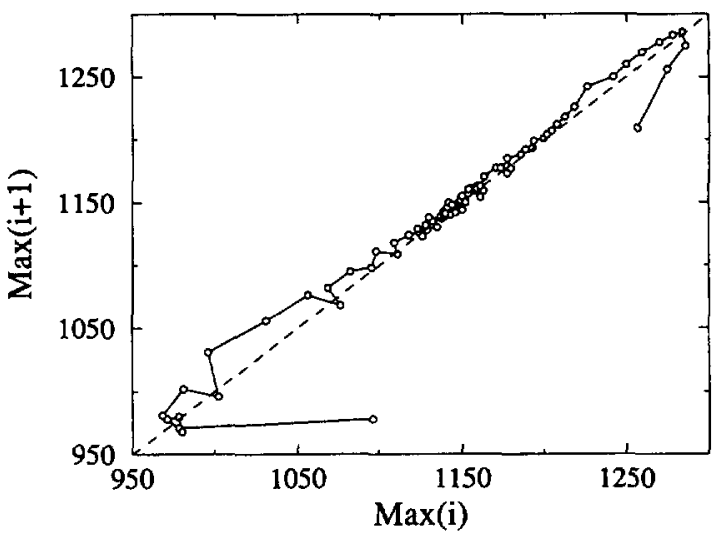

Fig, 19

Fig. 18. - Reconstruction of a 2D-attractor of type-I pseudo intermittency $(d=0.6 \mathrm{~mm}$ and $I=285 \mathrm{~mA}$ ).

Fig. 19. - First return map for the maximum of the time series. 


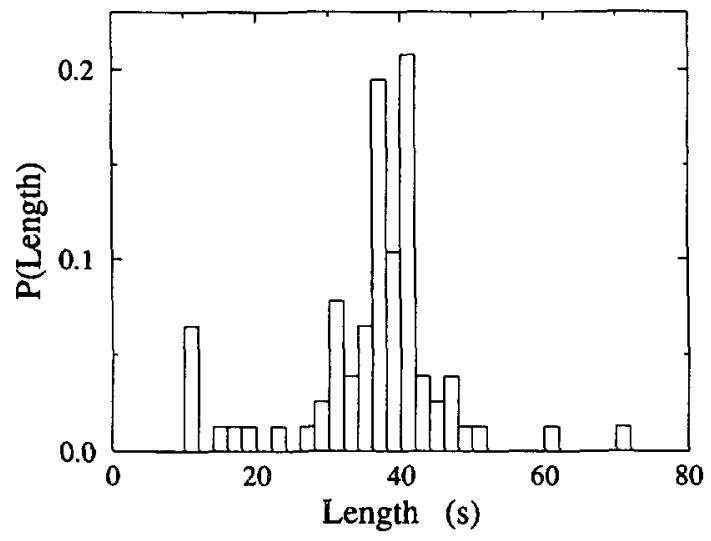

Fig. 20. - Relative probability distribution of the length of laminar phases.

Figure 21 displays the power spectrum evaluated by averaging 29 spectra built from 4096 data points. The broadening of the distribution around $40 \mathrm{~s}$ induces a spectral domain at small frequencies where we may write PSD $\propto F^{-a}$ The exponent $a$ is equal to 2.6 which is quite far from theoretical results where $a \approx 1$ [23]. Our experimental value cannot be compared with other experimental values, due to the lack of experimental intermittency spectra in the literature.

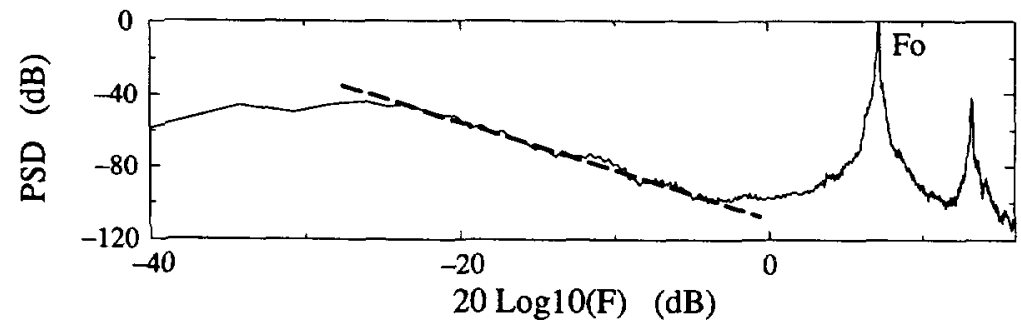

Fig. 21. - Average power spectrum density in logarithmic scales.

\section{Coexistence of attractors.}

Another classical feature of nonlinear systems is the coexistence of multiple attractors, each attractor being surrounded by its own basin of attraction. This behaviour occurs in hot-wire experiments, i.e. for a given parameter vector $(d, I)$, the state of the system is not necessarily unique.

Examples are provided by two experiments performed with a constant current $I=295 \mathrm{~mA}$. Each experiment starts with the wire located at $d_{d}$, far from the surface. Then $d$ is decreased step by step down to $d_{\mathrm{b}}$. Finally, $d$ is increased from $d_{\mathrm{b}}$ back to $d_{\mathrm{a}}$. At each step, the time series $\bar{T}(t)$ is recorded and the power spectrum is computed. The free surface spatial state is also observed, to possibly emphasize the relation between temporal behaviour $\bar{T}(t)$ and spatial behaviour of the waves. 
5.1 FIRST EXPERIMENT. - Here, $d_{\mathrm{a}}=3.0 \mathrm{~mm}$ and $d_{\mathrm{b}}=0.56 \mathrm{~mm}$. Three different cases may be distinguished.

(i) For $d$ ranging from 3.0 down to $0.94 \mathrm{~mm}$, all individual waves propagate in single direction, say from the right to the left, according to : $\leftarrow \leftarrow \leftarrow \leftarrow$.

(ii) For $d$ decreasing further down to $0.56 \mathrm{~mm}$, and also for $d$ increasing back from 0.56 up to $1.46 \mathrm{~mm}$, there is a sink point on the left part of the wire, according to the diagram :

(iii) Then, for $d$ increasing further up to $3.0 \mathrm{~mm}$, the waves propagate again in an single direction, but now from the left to the right $: \rightarrow \rightarrow \rightarrow \rightarrow$.

All signals are periodic. except near the minima of $A$ which are discussed in section 3 (figure not given). Therefore, it is observed that the presence of a sink does not necessarily induce a complicated behaviour. In particular, frequencies measured on both sides of the sink were identical and consequently the sink location was constant.

5.2 SECOND EXPERIMENT. - Here, $d_{\mathrm{a}}=2.5 \mathrm{~mm}$ and $d_{\mathrm{b}}=0.76 \mathrm{~mm}$. The square root of the total power $(A)$ versus $d$ is displayed in figure 22. Again, three domains can be distinguished.

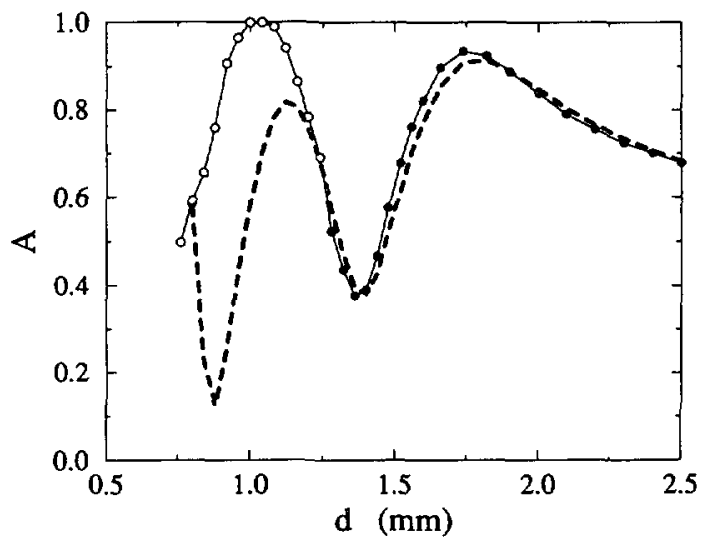

Fig. 22.- Evolution of $A=\sqrt{P}$ with $d$ showing three different domains for the second experiment.

(i) For $d$ ranging from 2.5 down to $0.84 \mathrm{~mm}$ (dashed line), the waves propagate from the left to the right $(\rightarrow \rightarrow \rightarrow \rightarrow)$ and signals are periodic.

(ii) For $d$ decreasing further down to $0.76 \mathrm{~mm}$ and then increasing back up to $1.24 \mathrm{~mm}$ (white circles), there is a sink in the right part of the wire $(\rightarrow \rightarrow \rightarrow \leftarrow)$ and signals are not periodic, as presently discussed.

(iii) Finally, for $d$ increasing again up to $2.5 \mathrm{~mm}$ (black circles), waves propagate again from the left to the right $(\rightarrow \rightarrow \rightarrow \rightarrow)$ and signals are periodic.

The appearance and disappearance of the sink therefore exhibit a strong hysteresis. The states of the system in phases (ii), i.e. in the presence of the sink, are rather complex as illustrated in figure 23 where attractors are reconstructed for $d=0.84$ (a), 0.88 (b), 0.92 (c), 0.96 (d), 1.00 (e) and $1.16 \mathrm{~mm}$ (f). Poincaré sections for each attractor are shown in figure 24 .

The evolution of the attractors from (a) to (f), and particularly from (a) to (d), shows a gradual simplification of the structure corresponding to the unfolding of the top-right dark zone and to the fact that the central part of the attractor becomes less and less visited. 


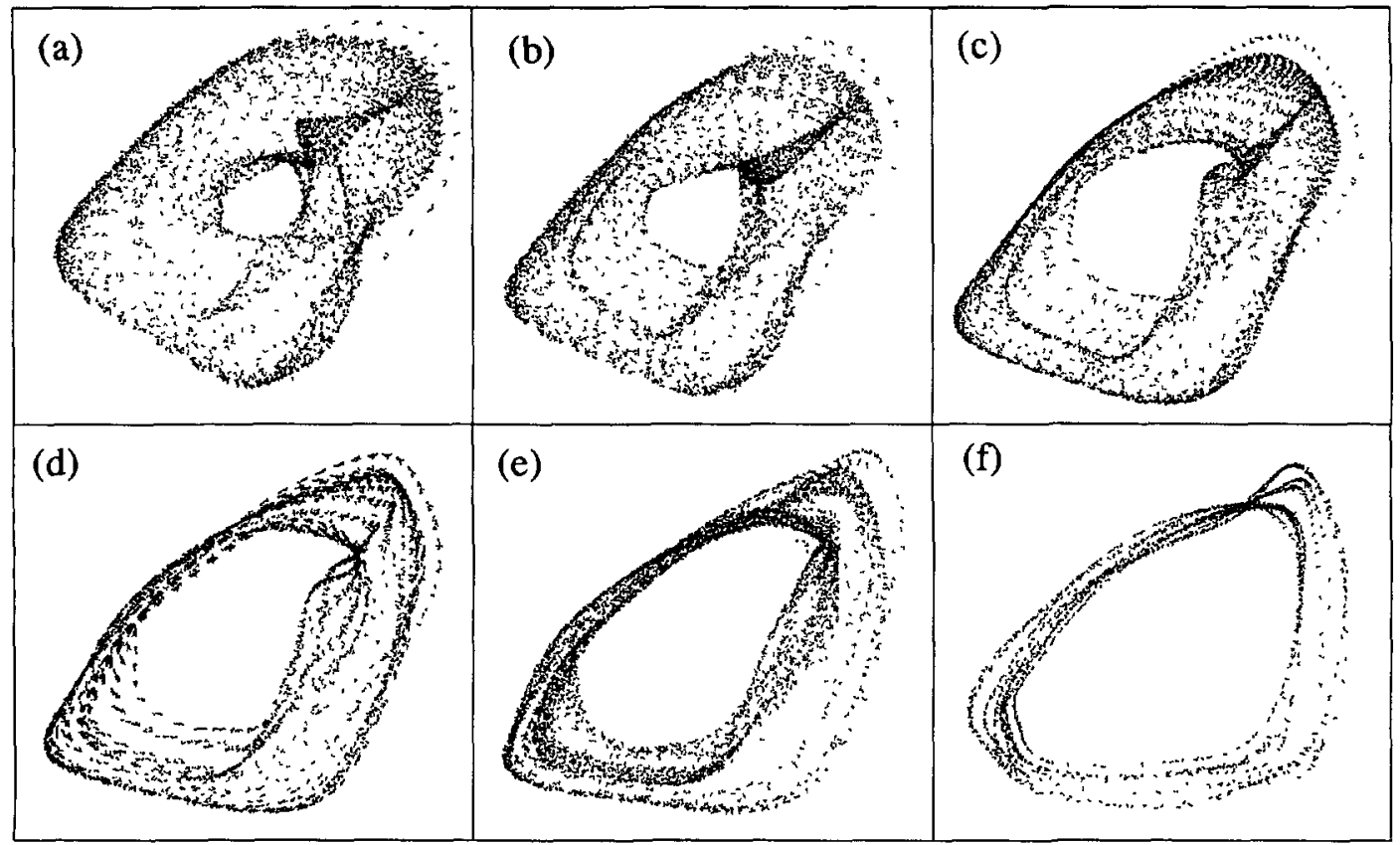

Fig. 23. - Reconstructed attractors for different values of $d$.

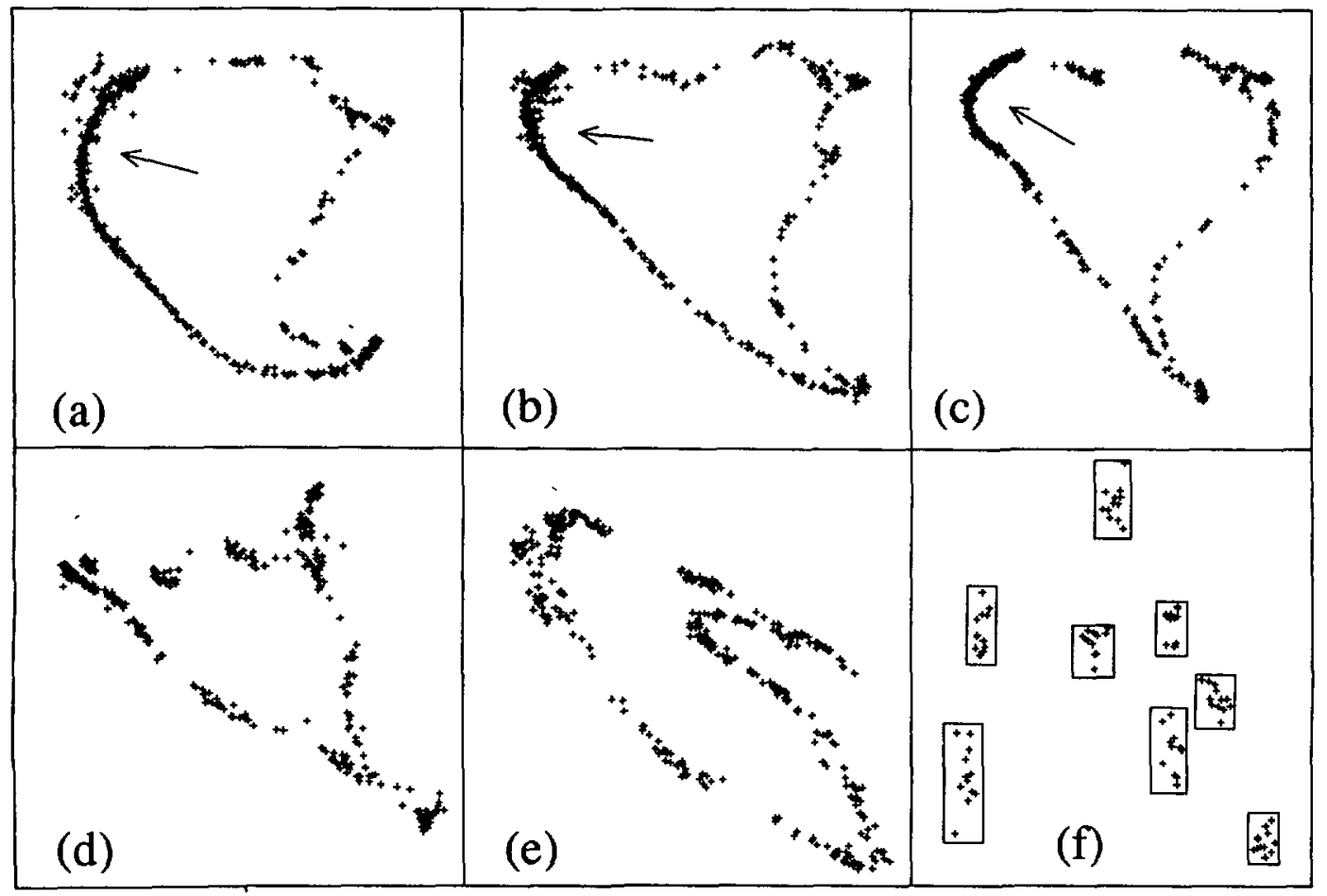

Fig. 24. - Poincaré sections of the attractors displayed in figure 23. 
Poincaré sections show that the attractors are essentially two-tori with a probability of presence evolving to a more discontinuous repartition from (a) to (f). In particular, in (f), all data points are contained in eight disconnected boxes.

In (a), we are faced with phase intermittency, the arrow pointing to a dark zone where the phase evolves slowly. The disappearance of phase intermittency may then be followed up to (f) where the presence of eight distinct domains corresponds to a frequency locking near the rational $7 / 8=0.875$. Indeed, the power spectrum then shows two basic frequencies $F_{1}$ and $F_{2}$ in the ratio $F_{1} / F_{2}=0.88$.

One also has to remark that the appearance and disappearance of the sink occur when the total power is close to a minimum. On both sides of the minimum, the number of individual waves is changed by a value of 1 .

Both experiments indeed show the presence of hysteresis connected with the coexistence of attractors. When the wire is far enough from the surface $(d \geqslant 2 \mathrm{~mm})$, we always found a single periodic state. On the other hand, when the wire is closer to the surface, multiple attractors may be present. For example, for $d=1.0 \mathrm{~mm}$, we may observe (i) a periodic state with all the waves propagating in the same direction (ii) a periodic state with a sink point or (iii) a quasiperiodic state with a sink point.

\section{Conclusion.}

New experimental results on instabilities produced when hot-wire heating a fluid below the free surface have been presented. In spite of the interaction of many phenomena (heat conduction and convection, Marangoni effect, wave propagation), the system can be reduced to a dynamical system with a small number of degrees of freedom. Some emphasis has been laid on the relations between temporal averaged temperature signals and wave spatial patterns along the wire. In the present case where only one defect at most is present in the train of waves, it has been demonstrated that there is a strong relationship between temporal and spatial behaviour. Many different kinds of behaviour may be observed and analyzed in a precise way, thanks to the very good Signal/Noise Ratio achieved, including quasiperiodicity or type-I and type-II intermittencies. A simple model enables us to predict in which situations complex behaviour may be expected. Interesting future investigations would include the study of hot-wire forced experiments in which the supplying current $I$ would be modulated. Experiments along a very long hot-wire allow the experimental study of spatio-temporal chaos. Also, the unsolved issue to understand the exact nature of the waves is warranted to be of utmost interest.

\section{References}

[1] Gouesbet G. and Lefort E., Dynamical states and bifurcations of a thermal lens using spectral analysıs. Phys. Rev. A 37 (1988) 4903-4915.

[2] Gouesbet G., Pashinin P. P., Rastopov S. F. and Sukhodolsky A. T., Oscillatory instability of liquids drops-lens by continuous laser heating, Sov. Phys. Lebedev Institute Rap. (USA) 11 (1991) 30-2 ; Translation of Sb. Kratk. Soobshch. Fiz. AN SSSR. Fiz. Inst. P.N. Lebedeva, Russia 11 (1991) 33-5.

[3] Meunier-Guttin-Cluzel S., Maheu B. and Gouesbet G.. Combined approaches and characterizations of experumental chaotic attractors in thermal lensing, Physica D 58 (1992) 423-440.

[4] Enokida Y. and Suzuki A., «Application of laser-induced thermal lens oscillation to concentration control in solvent extraction processes ". Proceedings of the International Symposium: Instabilities in multiphase flows, Rouen, May 11 th-14th, 1992. G. Gouesber, A. Berlemont, Eds. (Plenum Publishing Corporation, 1993). 
[5] Ringuet E. Rozé C. and Gouesbet G., Experimental observation of type-I and type II intermittencies, Proceedings of the International Symposium : Instabilities in multiphase flows, Rouen, May 11 th-14th, 1992. G. Gouesbet, A. Berlemont, Eds. (Plenum Publishing Corporation, 1993).

[6] Rozé C., Gouesbet G. and Darrıgo R., Oscillatory instabilıties produced by temperature-controlled hot-wire heating below an interface, I Fluid Mech 250 (1993) 253-276.

[7] Ringuet E., Rozé C. and Gouesbet G., Experimental observation of type-II intermittency in a hydrodynamic system, Phys. Rev. E 47 (1993) 1405-1407.

[8] Vince J. M. and Dubois M.. Hot-wire below the free surface of a liquid : structural and dynamical properties of a secondary instability, Europhvs. Lett. 20 (1992) 505-510.

[9] Dubors M., Daviaud F., Ronsin O. and Bergé P., Travelling waves in pure fluids locally heated along wires, in «New Trends in Nonlinear Dynamics : Non-variational Aspects», C. PerezGarcia Ed., Physica D 61 (1992) 140.

[10] Burguete J., Mancini H. L. and Pérez-Garcia, Dynamics of a secondary instability in BénardMarangoni convection with unidimensionnal heating. Europhys. Lett. 23 (1993) 401-407.

[11] Paranthoën P. and Petit C.. Influence de la conduction entre le capteur et ses supports sur la mesure des fluctuations de température dans un écoulement turbulent effectué à l'aide d'un thermomètre à résistance, Lett. Heat Mass Tiansfer 6 (1979) 311.

[12] Reıman J., Experimental investigaton of free convection flow from wires in the vicinity of phase interfaces, Int. J Heat Mass transfer 17 (1973) 1051.

[13] Weill M. E., Rhazi M. and Gouesbet G., Experimental investigation of oscillatory phenomena produced by a hot-wire located near and below a free surface, $J$ Phys. France 46 (1985) 1501.

[14] Maquet J., Gouesbet G. and Berlemont A., Numerical simulation of surface tension and combined buoyancy driven convection in a liquid layer heated by a hot-wire, Int. J. Mass Transfer 35 (1992) 2695-2703.

[15] Gouesbet G.. Simple model for bıfurcations ranging up to chaos in thermal lens oscillations and associated phenomena, Phys. Rev. A 42 (1990) 5928-5945.

[16] Kraenkel R. A.. Pereira J. G. and Manna M. A.. Hydrothermal surface-wave instability and the Kuramoto-Sivashinsky equation, private communication.

[17] Roux J. C., Simoyi R. H. and Swinney L., Observation of a strange attractor, Physica D 8 (1983) 257-266.

[18] Bergé P. and Dubors M., Intermittence de phase au voisinage d'un accrochage de fréquences : une route vers le chaos? J. Phys. Lett. France 46 (1985) L431-L436.

[19] Rosenstein M. T., Collins J. J. and De Luca C. J., A practical method for calculating largest Lyapunov exponents from small data sets, Physica D 65 (1993) 117-134.

[20] Pomeau Y. and Manneville P., Intermittent transition to turbulence in dissipative dynamical system. Commun. Math. Phys. 74 (1980) 189-197.

[21] Ringuet E., Rozé C. and Gouesbet G., «Various behaviours obtained by heating a fluid below its free surface with a hot-wire », International symposium on spatio-temporal structure and chaos in heat and mass transfer process, Athens, Greece, May 18-22, 1992. Proceedings published by the Int. Center Heat Mass Transfer (1992) 1-11.

[22] Hirsch J. E., Huberman B. A. and Scalapino D. J., Theory of intermittency, Phys. Rev. A 25 (1982) 519-532.

[23] Mori N.. Kurokı S. and Mori H., Power spectra of intermittent chaos due to the collapse of period-3 window, Prog Theor Phys 79 (1988) 1260-1264. 